\title{
MODULATORY ACTIVITY OF BEE POLLEN AGAINST THE TOXICITY OF ANTITUBERCULOSIS DRUGS RIFAMPICIN AND ISONIAZID IN TESTIS OF SPRAGUE DAWLEY RATS
}

\author{
UMESH BHARTI ${ }^{1 *}$, NEELIMA R KUMAR ${ }^{2}$, JASPREET KAUR ${ }^{3}$ \\ ${ }^{1}$ Department of Zoology, Government College for Girls, Chandigarh, India. ${ }^{2}$ Department of Zoology, Panjab University, Chandigarh, India. \\ ${ }^{3}$ Department of Biotechnology, UIET, Panjab University, Chandigarh, India. Email: dbhv90@gmail.com
}

Received: 08 May 2017, Revised and Accepted: 25 May 2017

\section{ABSTRACT}

Objective: The aim of this study is assessment of protective role of bee pollen in antituberculosis drug (rifampicin and isoniazid)-induced toxicity in testis of Sprague Dawley rats.

Methods: Healthy rats weighing $180 \pm 20$ g were selected for the study. Rats were divided into five groups, i.e., Group A (control), Group B (100 mg/kg body weight/day rifampicin-treated), Group C (rifampicin $100 \mathrm{mg} / \mathrm{kg}$ body weight and bee pollen $100 \mathrm{mg} / \mathrm{kg}$ body weight), Group D (isoniazid $50 \mathrm{mg} / \mathrm{kg}$ body/day treated), and Group E (isoniazid $50 \mathrm{mg} / \mathrm{kg}$ body weight/day with bee pollen $100 \mathrm{mg} / \mathrm{kg}$ body weight/day) serve as experimental groups.

Results: Aqueous extract of bee pollen when administered along with the antituberculosis drugs (rifampicin, isoniazid) showed significant reduction in the level of malondialdehyde while the activity of superoxide dismutase, glutathione (GSH) reductase, glutathione peroxidase, glutathioneStransferase, catalase, and GSH was elevated representing the antioxidant potential of bee pollen against the drug-treated groups. Supplementation of bee pollen significantly reduced histological changes in the testis of drug-induced groups such as smaller epithelial height, germ cell loss, and irregular seminiferous tubules to near normal.

Conclusion: Bee pollen has shown the modulatory effect against damage and oxidative stress induced by antituberculosis drugs (rifampicin and isoniazid) in rat testis.

Keywords: Bee pollen, Testis, Histology, Oxidative stress, Rifampicin, Isoniazid.

(c) 2017 The Authors. Published by Innovare Academic Sciences Pvt Ltd. This is an open access article under the CC BY license (http://creativecommons. org/licenses/by/4. 0/) DOI: http://dx.doi.org/10.22159/ajpcr.2017.v10i9.19701

\section{INTRODUCTION}

The World Health Organization (WHO) has shown concern about the burden of tuberculosis in the developing countries. About 1.8 million deaths are caused by Mycobacterium tuberculosis worldwide and there is an increase in a number of new cases as well [1]. Even though rifampicin and isoniazid are wonder drugs in effective management of tuberculosis, certain toxic effects have been documented in humans. The coadministration of ethambutol, isoniazid, rifampicin, and pyrazinamide to male rats during the period of spermatogenesis caused an increase in the level of malondialdehyde (MDA), and the rate of glutathione (GSH) and protein SH-group contents was significantly decreased [2]. Fatal decrease of male fertilizing capacity and fertility was also reported by antituberculosis drugs. To minimize the adverse effects of tuberculosis therapy, there is a necessity to supplement tuberculosis therapy with natural products. Various natural products such as Ocimum sanctum [3], garlic [4], Silymarin [5], Aloe vera [6], and propolis [7] had potential to reduce the oxidative stress caused by antituberculosis drugs. Keeping all these activities in mind, the present experiment was designed to study the preventive role of bee pollen against toxic effect of antituberculosis drug induced toxicity in Sprague Dawley (SD) rats.

\section{MATERIALS AND METHODS}

Drugs

Rifampicin and Isoniazid drugs were purchased from HiMedia.

\section{Bee pollen}

Bee pollen was collected from Langstroth beehives maintained in an apiary at Majri village near old Panchkula, Haryana. Aqueous extract of bee pollen was prepared.

\section{Experimental animals}

Male SD rats of body weight in the range of $180 \pm 20$ g were obtained from the central animal house of Panjab University, Chandigarh. All experiments and protocols reported here strictly followed the principle as laid down by the Institutional Animal Ethical Committee (IAEC), Panjab University, Chandigarh vide letter no PU/IAEC/2013/18.

\section{Experimental protocol}

The rats were divided into five groups randomly $(n=6)$

- Group A received only the normal diet.

- Group B received along with normal diet received the rifampicin (100 mg/kg body weight/day)

- Group C received along with normal diet rifampicin $(100 \mathrm{mg} / \mathrm{kg}$ body weight/day) and bee pollen (100 mg/kg body weight/day)

- Group D received along with normal diet isoniazid (50 mg/kg/day)

- Group E along with normal diet received with isoniazid (50 mg/kg/day) and bee pollen $(100 \mathrm{mg} / \mathrm{kg} /$ day $)$.

These treatments were given daily for 30 days. All rats were kept under identical conditions for 30 days with free access to food (standard laboratory pellet diet from Ashirwaad Industries) and water. After the last treatment, rats were sacrificed under light anesthesia and cervical dislocation. Testes were removed and weighed for preparing tissue homogenate. Testis samples were rapidly excised; after dissection, washed with ice-cold saline and 10\% testis homogenates were prepared in ice-cold phosphate-buffered saline (PBS, pH 7.4) using homogenizer for 2 minutes at 3000 r.p.m in ice till total disruption of cells. Post-mitochondrial supernatant was prepared by centrifugation at $10,000 \mathrm{~g}$ for 20 minutes at $4^{\circ} \mathrm{C}$ in cold centrifuge. 


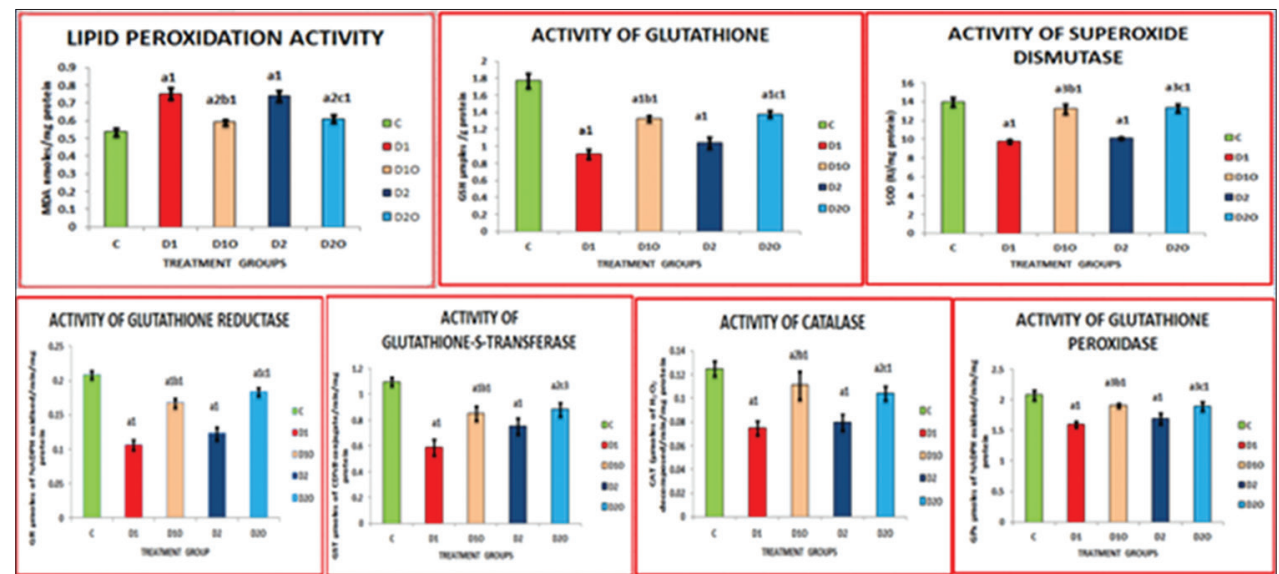

Plate 1: Oxidative stress parameters in testis of SD rat. C: Control, D1: Ripfampicin, D10: Ripfampicin+Pollen, D2: Isoniazid,

D20: Isoniazid+Pollen as treatment groups. $p$ value: $a 1(p \leq 0.001), a 2(p \leq 0.001)$, a3: $(p \leq 0.05)$ statistically significant difference w.r.t. control. $p$ value: b1 ( $\leq 0.001), b 2(p \leq 0.001), b 3:(p \leq 0.05)$ statistically significant difference w.r.t. Ripfampicin treated group. $P$ value: $c 1$ ( $\leq \leq 0.001), c 2$ ( $\leq \leq 0.001), c 3:(p \leq 0.05)$ statistically significant difference w.r.t. Isoniazid treated group

\section{Biochemical analysis}

Lipid peroxidation product was determined by measuring MDA content in tissue homogenates according to the method of Begue and Aust [8] spectrophotometrically at $532 \mathrm{~nm}$. Superoxide dismutase (SOD) activity was determined by following the method of Kono [9] at $560 \mathrm{~nm}$. Catalase (CAT) activity was determined according to method of Luck [10] based on determination of the $\mathrm{H}_{2} \mathrm{O}_{2}$ decomposition rate at $240 \mathrm{~nm}$. The level of reduced GSH was measured as protein-free sulfhydryl content by the method of Sedlak and Lindsay [11] at $412 \mathrm{~nm}$, activity of glutathione S-transferase (GST) was determined using spectrophotometer by the method of Habig et al.[12] at $340 \mathrm{~nm}$, glutathione peroxidase (GSH-Px) was assessed according to the method of Pagila and Valentine [13] at $340 \mathrm{~nm}$, and glutathione reductase (GR) activity was determined by the method of Carlberg and Mannervick [14].

\section{Histopathologic analysis}

Testis tissues from the rats were fixed in $10 \%$ formalin solution overnight. After fixation, the sections were subjected to routine histologic tissue preparation and dehydrated and embedded in paraffin. Paraffin blocks were sliced to $0.5 \mathrm{~mm}$ thickness with a microtome and after hematoxylin and eosin staining, and they were analyzed under a light microscope

\section{Statistical analysis}

All data were expressed as the mean \pm standard deviation. Data were analyzed by one-way analysis of variance and means were compared. A probability value of $\mathrm{p} \leq 0.05$ was considered statistically significant.

\section{Oxidative stress-related parameters}

Generation of ROS on administration of antituberculosis drugs (rifampicin and isoniazid) leads a series of imbalances measured in terms of related enzymes and molecules in rat testis PMS. The level of lipid peroxides was assessed by estimation of formation of MDA, an indicator of lipid peroxidation. Animals treated with rifampicin $(\mathrm{p}<0.001)$ and isoniazid $(\mathrm{p}<0.001)$ showed a significant increase in the level of MDA concentration compared to control group $(0.54 \pm 0.02$ nmoles/mg protein). Administration of bee pollen extract [15] with antituberculosis drugs significantly reduced the adverse effect by $30.59 \%$ (rifampicin) and $41.24 \%$ (isoniazid) on testis lipid peroxidation (Plate 1).

The activity of SOD significantly decreased in rifampicin $(9.763 \pm 0.214 \mathrm{IU} / \mathrm{mg}$ protein)-treated rats and in isoniazid (10.165 $\pm 0.199 \mathrm{IU} / \mathrm{mg}$ protein)-treated rats showing the depletion of antioxidant system. In rifampicin- and bee pollen-treated rats, values were $43.8 \%(14.038 \pm 0.211 \mathrm{IU} / \mathrm{mg}$ protein) while in isoniazid- and bee pollen-treated group, the values were $36.9 \%$

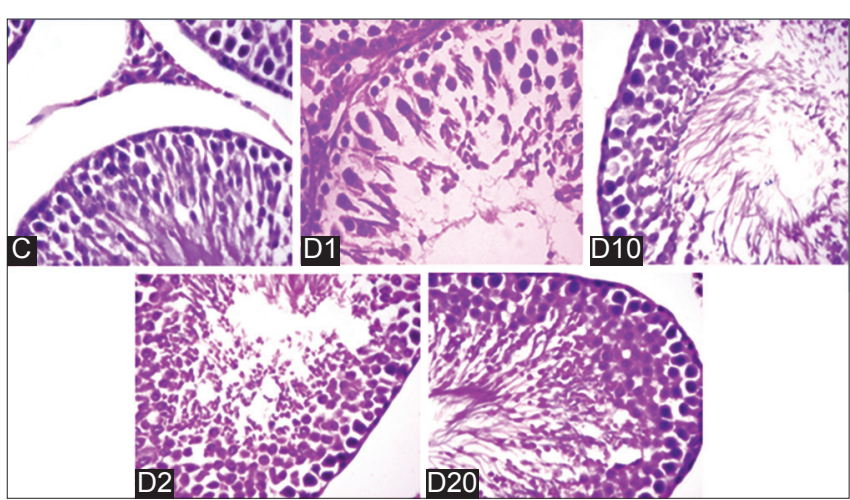

Plate 2: Histopathology of testis. C: Control, showing normal cells in seminiferous tubule. D1: Rifampicin, the number of spermatocytes and spermatogonia decreased.

D10: Rifampicin+Pollen, seminiferous tubules with several layers of epithelial cells and recovery of germ cell population.

D2: Isoniazid, the germinal epithelium eroded. D20: Isoniazid+Pollen, thickness of germinal epithelium improved $(\times 40)$

higher (13.915 $\pm 0.156 \mathrm{IU} / \mathrm{mg}$ protein) as compared to rifampicin- and isoniazid-treated groups, respectively. These values were significantly near the control values of $(13.835 \pm 0.176 \mathrm{IU} / \mathrm{mg}$ protein). Decreased activity of CAT $40 \%$ (rifampicin-treated rats) and 36\% (isoniazidtreated rats) was found in rat testis supernatants as compared to control group $\left(0.125 \pm 0.006 \mu\right.$ moles of $\mathrm{H}_{2} \mathrm{O}_{2}$ decomposed $/ \mathrm{min} / \mathrm{mg}$ protein) indicating attenuation of the most significant antioxidant enzyme. Significant increase $(\mathrm{p}<0.001)$ was recorded when bee pollen was administered along with antituberculosis drugs indicating positive effect on CAT activity. Similarly, treatment of rats with rifampicin and isoniazid caused a significant decrease $(\mathrm{p}<0.001)$ in reduced GSH level, GST when compared with control group. GSH (45.51\% and 32.44\%) and GST $(45.07 \%$ and $16.95 \%)$ activity improved on bee pollen cotreatment with rifampicin and isoniazid, respectively. GR and GPx activities were significantly elevated in bee pollen-treated groups $(p<0.001)$ in comparison to rifampicin- and isoniazid-treated group that produced a significant decrease in the activities of GR and GPx $(\mathrm{p}<0.001)$.

\section{Histopathological study of testis}

Under Nikon phase-contrast microscope, examination of testis tissue in control group revealed Plate 2 - C, the presence of healthy blood vessels with normal architecture of seminiferous tubules. The spermatogonia, spermatocyte, spermatids, spermatozoa, and Sertoli cells can be easily 
identified in the seminiferous tubule. The lumen of seminiferous tubule is occupied by large number of spermatozoa. Leydig cells are also clearly seen in the interstitial spaces. In Rifampicin (100 mg/kg body weight)treated group, the histopathology showed distorted morphology in contrast to that in the control group. The seminiferous tubules were shrunken and with wavy outlines. A number of spermatocytes and spermatogonia are decreased. Debris of dead cells can be seen in the lumen of seminiferous tubules. Distorted and less concentration of germ cells was recorded in groups treated with rifampicin drug. Detachment of spermatogenic cells was also recorded Plate 2 - D1. In Isoniazid (50 $\mathrm{mg} / \mathrm{kg}$ body weight)-treated group, the histopathology showed anomalous morphology in contrast to that in the control group (Plate 2 - D2. The germinal epithelium was eroded. Severe interstitial edema was seen. Multinucleate giant cells were frequently observed and reduced numbers of spermatozoa were present

Aqueous extract of bee pollen when fed along with drugs revealed noticeable enhancement in the testis histoarchitecture and clogged the rifampicin-/isoniazid-induced alterations as shown in Plate 2 - D10, D20. The seminiferous tubules consist of several layers of epithelial cells with recovery of germ cell population was seen in the lumen. The thickness of germinal epithelium was also improved with treatment with bee pollen. Morais et al. (2011) advocated that bee pollen constitutes a good source of healthy compounds and is a potential source of new antimicrobial agents. Shayakhmetova et al. reported that the rats groups administered with ethambutol, rifampicin, isoniazid, and pyrazinamide showed increased level of germinal epithelium exfoliation into the lumen of seminiferous tubules. Reports of previous workers supported the presence of various antioxidants in bee pollen, and the present study supports the protective efficacy of bee pollen against antituberculosis drug-induced toxicity. The present study attempts to create awareness concerning the modulatory potential of bee pollen against antituberculosis drug-induced toxicity in SD rats.

\section{DISCUSSION}

Reactive oxygen species (ROS) play a vital role in sperm maturation and capacity. The delicate equilibrium between ROS production and recycling is crucial for normal spermatogenesis and its excessive generation causes male infertility [2]. Bee pollen has arisen as a promising agent with the recognition of its antioxidant effects. The bee pollen contained about $67.7 \%$ carbohydrates, $21.8 \%$ crude protein, $5.2 \%$ crude fat, and $2.9 \%$ ash. The phenolic contents $(12.9$ to $19.8 \mathrm{mg}$ of gallic acid equivalents/g of extract) and flavonoid contents (4.5-7.1 mg of catechin equivalents/g of extract) were reported from the samples. About $100 \mathrm{~g}$ of bee pollen contained about 396.4-411.1 kcal energy [16]. Rifampicin- and isoniazid-induced toxicity and oxidative stress relationship have been reported previously $[3,4,7]$. Mechanism of drug toxicity is through production of free radical and their capability to induce apoptosis by the production of ROS, lipid peroxidation, and cell membrane damage $[5,6]$. In the present study, statistically significant differences were observed in the oxidative stress markers in testis tissues and in histopathological parameters of male SD rats treated with rifampicin and isoniazid as compared to control group rats, and also statistically significant amelioration of toxicity was found in rifampicin and isoniazid plus bee pollen-treated group as compared to antituberculosis drugs alone. The results suggested that when administered at the indicated dose and for the given period to rats, bee pollen caused modulatory influence on the parameters investigated. Bee pollen has been found to have some protective effect against carbon tetrachloride-induced toxicity [17] and malathioninduced toxicity [18]. Previous studies showed that antituberculosis drugs damaged the endogenous antioxidant system of the whole body [3-7] In our results, it is clearly reflected that supplementation of diet with bee pollen in animals has antioxidant and protective effect when given along with antituberculosis drugs (rifampicin as well as isoniazid). It is thus observed that bee pollen has strong potential to provide protection against antituberculosis drugs-induced toxicity in rats as evidenced by improved histological parameters and rebuilding of the antioxidant/oxidative status.

\section{REFERENCES}

1. WHO. Global Tuberculosis Report 2016. Geneva: WHO; 2016.

2. Shayakhmetova GM, Bondarenko LB, Kovalenko VM. Damage of testicular cell macromolecules and reproductive capacity of male rats following co-administration of ethambutol, rifampicin, isoniazid and pyrazinamide. Interdiscip Toxicol 2012;5(1):9-14.

3. Ubaid RS, Anantrao KM, Jaju JB, Mateenuddin M. Effect of Ocimum sanctum (OS) leaf extract on hepatotoxicity induced by antitubercular drugs in rats. Indian J Physiol Pharmacol 2003;47(4):465-70.

4. Pal R, Vaiphei K, Sikander A, Singh K, Rana SV. Effect of garlic on isoniazid and rifampicin-induced hepatic injury in rats. World $\mathrm{J}$ Gastroenterol 2006;12(4):636-9.

5. Eminzade S, Uras F, Izzettin FV. Silymarin protects liver against toxic effects of anti-tuberculosis drugs in experimental animals. Nutr Metab 2008;5:18

6. Zodape GV, Bhise PP. Effect of Aloe vera extract on the hepatotoxicity induced by isoniazid and rifampicin drug in male wistar rats. Int $\mathrm{J}$ Pharm Sci Res 2016;7(3):1314-20.

7. Bharti U, Kumar NR, Kaur J. Protective effect of bee propolis against anti-tuberculosis drugs (rifampicin and isoniazid) induced haematological toxicity in Sprague Dawley rats. Asian J Pharm Clin Res 2017;10(3):188-90.

8. Begue JA, Aust SD. Microsomal lipid peroxidation. Methods Enzymol 1978;52:302-10.

9. Kono Y. Generation of superoxide radical during autoxidation of hydroxylamine and an assay for superoxide dismutase. Arch Biochem Biophys 1978; 186:189-95.

10. Luck H. Estimation of catalase activity. In: Bergmeyer U, editor. Methods of Enzymology. New York: Academic Press; 1974. p. 885.

11. Sedlak J, Lindsay RH. Estimation of total protein bound and nonprotein sulfhydryl groups in tissue with Ellmann's reagent. Anal Biochem 1968;25(1):192-205

12. Habig WH, Pabst MJ, Jakoby WB. Glutathione-S-transferases. The first enzymatic step in mercapturic acid formation. J Biol Chem 1974;249(22):7130-9.

13. Pagila DE, Valentine WN. Studies on the quantitative and qualitative characterization of erythrocyte glutathione peroxidase. J Lab Clin Med 1967;70(1):158-69.

14. Carlberg I, Mannervick B. Purification and characterization of the flavoenzyme glutathione reductase from rat liver. J Biol Chem $1975 ; 250(14): 5475-80$

15. Yamaguchi M, Hamamoto R, Uchiyama S, Ishiyama K, Hashimoto K. Preventive effects of bee pollen Cistus ladaniferus extract on bone loss in streptozotocin-diabetic rats in vivo. J Health Sci 2007;53(2):190-5.

16. Feás X, Vázquez-Tato MP, Estevinho IL, Seijas JA, Iglesias A. Organic bee pollen: Botanical origin, nutritional value, bioactive compounds. Antioxidant activity and microbiological quality. Molecules 2012;17(7):8359-77.

17. Yildiz O, Can Z, Saral O, Yulug E, Ozturk F, Aliyazicioglu R, et al. Hepatoprotective potential of chestnut bee pollen on carbon tetrachloride-induced hepatic damages in rats. Evid Based Complement Alternat Med 2013;2013:1-9.

18. Kandiel MM, El-Asely AM, Radwan HA, Abbass AA. Modulation of genotoxicity and endocrine disruptive effects of malathion by dietary honeybee pollen and propolis in Nile tilapia (Oreochromis niloticus). J Adv Res 2014;15(6):671-84. 